\title{
A CENTRIFUGAL FILTER FOR STERILIZING SMALL AMOUNTS OF FLUID
}

\author{
BY \\ G. R. F. HILSON AND S. D. ELEK \\ From the Department of Bacteriology, St. George's Hospital Medical School, London
}

(RECEIVED FOR PUBliCATION AUGUST 23, 1949)

It is often desirable in routine and experimental laboratory work to obtain sterile filtrates of small quantities (1-5 ml.) of liquid, and the principal disadvantage of the usual types of bacterial filter is the relatively large loss by absorption in the filtering element. To overcome this difficulty Morton and Czarnetzky (1937) used a funnel fitted with a sintered (fritted) glass disk of $30 \mathrm{~mm}$. diameter, porosity " 5 auf 3," the capacity being $30 \mathrm{ml}$. This funnel was fitted with a rubber stopper into the mouth of a large-bore test-tube having a side-arm to which suction was applied. Asheshov (1938) described a smaller piece of apparatus consisting of a small Chamberland $L_{3}$ or $L_{5}$ filter-candle fitted to the lower end of a $10-20 \mathrm{ml}$. pipette which had a rubber bulb or suction lead at its upper end. The candle was immersed in the fluid to be sterilized and suction applied.

The filtration pressure resulting from centrifugation may be used in a clarifying and sterilizing filter, and Boerner (1942) described a metal filter for use with ordinary laboratory centrifuges. This is made up of two parts: a base holding a Seitz filter pad, and a cylindrical upper part threaded at its lower end. It is assembled by screwing down the top cylinder tightly on to the filter pad and the filter is then fitted into the top of a standard metal centrifuge tube and spun at 2,000 revolutions per minute. When filled to its capacity of $2 \mathrm{ml}$. with, for example, serum, filtration is complete in 10 to 15 minutes with a loss of about $0.3 \mathrm{ml}$. (15\%). Less viscid fluids filter more rapidly and the loss is smaller. This filter is a marked advance, in principle and design, on the two types mentioned above, and the loss of fluid is minimal. There are, however, certain disadvantages: the filter and its container are sterilized apart, with the risk of contamination; a direct rotational shearing force is applied to the filter pad, with the likelihood of a disturbance of structure and unreliability in use ; and the capacity is too small in relation to the filtration area and overall dimensions.

The filter described here (see Fig. 1) follows that of Boerner in principle, and consists essentially of a flanged metal tube with its upper part separated by a Seitz EK pad from the lower, the overall dimensions allowing of its use in standard laboratory centrifuges. In some centrifuges an adapter collar may be required to prevent the filter from touching the outer casing. The upper portion, or cup, into which the fluid to be filtered is placed has a capacity of $5 \mathrm{ml}$. It has a flange like an ordinary centrifuge bucket and a pair of facets on which a spanner may grip. Its lower part carries an outer thread and tapers to a pressure ring, which, when the filter is assembled, 


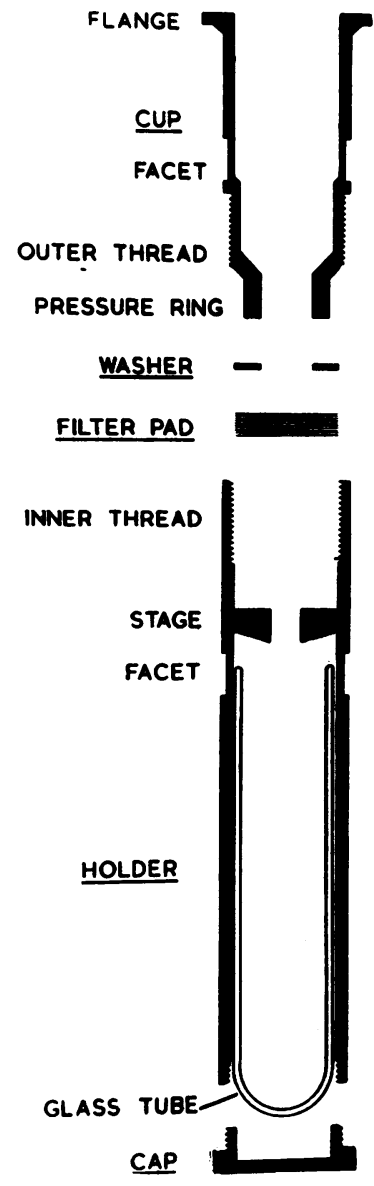

FIG. 1.-Diagram of the filter, the actual measurements of which are $4 \frac{1}{2}$ in. high by $\frac{3}{4}$ in. diameter.

is separated from the filter pad by a metal washer. The lower section of the apparatus has at its upper end a deep internal thread, a similar pair of facets for a spanner, and a stage on which the filter pad rests. The stage has a hole through the centre so that the drops of filtrate are directed down the central axis. Below the stage the holder contains a glass tube to receive the filtrate and is closed at its lower end by a screw cap knurled for tightening by hand.

In use, the holder is first loaded with the glass receiving tube, and the filter pad is placed on the stage, followed by the washer. If pads of suitable size are not available, they may be cut out of larger well-tested Seitz filter pads. With the aid of a pair of spanners the cup is then screwed down firmly into the top of the holder, thus tightly compressing the pad between the stage and the washer. The leverage of the spanners is needed to give a greater force than that obtained with a milled surface by hand. The assembled filters are sterilized in pairs in the autoclave. Filtration is complete in 15 to 25 minutes at about 2,500 r.p.m., depending on the viscosity and volume of the fluid, and the loss by absorption is not more than $0.4 \mathrm{ml}$. (8\% of $5 \mathrm{ml}$.). For safety, the cup may be corked with a rubber stopper without loss of efficiency.

This design was found reliable and handy. Its advantages are (a) that the rotational shearing strain communicated to the pad is negligible, since the pressure ring turns freely on the smooth upper surface of the washer, and the reliability of the pad is not endangered ; (b) the avoidance of the risk of contamination of the receiver, since the apparatus is made up and sterilized as a unit ; and $(c)$ the fluid loss is minimal.

The versatility of the filter is increased by the provision of a base with a side-arm for suction, on to which the uncapped holder may be screwed at an airtight joint, so converting the filter from the centrifugal to the normal suction type. Used in this way, a narrower, longer glass tube receives the filtrate, and the total capacity is increased.

\section{Summary}

A centrifugal bacterial filter is described, suitable for small quantities of fluid, and possessing the advantages of minimal percentage loss by absorption, ease of handling, avoidance of contamination of the filtrate, and convertibility to a suction type of filter of slightly larger capacity. S.W.1.

The filter is obtainable from Messrs. M. S. E., Ltd., 14, Spenser Street, London,

\section{REFERENCES}

Asheshov, I. N. (1938). J. Bact., 36, 197.

Boerner, Fred (1942). Amer. J. clin. Path., 12 (Tech. Sect.), 68.

Morton, H. E., and Czarnetzky, E. J. (1937). J. Bact., 34, 461. 